\title{
Forms of Structuralism: Bourbaki and the Philosophers
}

\author{
Jean-Pierre Marquis* \\ Département de philosophie \\ Université de Montréal \\ Montréal, Canada \\ Jean-Pierre.Marquis@umontreal.ca
}

\begin{abstract}
In this paper, we argue that, contrary to the view held by most philosophers of mathematics, Bourbaki's technical conception of mathematical structuralism is relevant to philosophy of mathematics. In fact, we believe that Bourbaki has captured the core of any mathematical structuralism.
\end{abstract}

\section{Introduction}

In the recent philosophical literature on mathematical structuralism, it is often declared that there is a sharp separation between the philosophical brand of mathematical structuralism and what mathematicians take to be structuralism. The standard position is that mathematicians practice a form of methodological structuralism and that the latter has very little to offer to philosophical discussions regarding the semantics, the ontology and the epistemology of structuralism. Ian Hacking, for instance, articulates this position explicitly in his book on philosophy of mathematics:

One of the most vigorous current philosophies of mathematics is named structuralism, but it has only a loose connection with the mathematician's structuralism of which we have spoken. (...) No label fit well, so let call structuralism of the Bourbaki type mathematician's structuralism, and that of recent analytic philosophy philosopher's structuralism. ([17, 237]

*The author gratefully acknowledge the financial support of the SSHRC of Canada while this work was done. This paper is part of a larger project on Bourbaki and structuralism which would not have seen the day without Michael Makkai's influence and generosity. I want to thank him for the numerous discussions we had on the subject. I also want to thank Alberto Perruzzi and Silvano Zipoli for inviting me to present this work at the conference on Bourbaki, mother structures and category theory. 
In his book Rigor and Structure, John Burgess arrives at the same conclusion:

... this [Bourbaki] kind of "structuralism" is now widely regarded as a trivial truism, so long as it is separated from the particulars of Bourbaki's attempt to pin down the relevant notion of "structure" in a technical definition. The contentious issue debated under the heading "structuralism" in contemporary philosophy of mathematics is not to be confused with Bourbaki's claim. $[4,17]$

In this paper, I argue that, on the contrary, once mathematical structuralism is properly understood and developed, that is when an adequate metamathematical analysis is provided, it ought to serve as a springboard to any philosophical discussion of mathematical structuralism. Let me be clear: I am not saying that the practice of mathematicians is directly relevant to philosophical issues tied to structuralism (although, the practice certainly cannot be ignored altogether by philosophers). Rather, I claim that Bourbaki's technical analysis and general views on mathematics include essential components illuminating the very nature of mathematical structuralism and should guide thinking on philosophical issues springing from structuralism. Bourbaki's analysis contains the main elements of the metamathematical analysis required, but it has unfortunately been misunderstood and misread. Surprisingly perhaps and as I will briefly indicate, Bourbaki himself is partly to blame for this situation.

The paper is organized as follows. In the first part, we take a quick look at how the philosophical literature has treated Bourbaki's structuralism and why it has been dismissed as being philosophically irrelevant. In the second part, we go back to Bourbaki's paper The Architecture of Mathematics, which is a popular exposition of Bourbaki's position on mathematics and, in particular, on mathematical structuralism and present its main theses. We believe that some of the central claims made by Bourbaki have been forgotten and that they can easily be extended to the contemporary mathematical context. We then move to Bourbaki's technical discussion of the notion of mathematical structure. In this section, our goal is to clarify the technical notion, in particular to emphasize its metamathematical character. In the last section, we sketch how the work by the logician Michael Makkai, namely First-Order Logic with Dependent Sorts, FOLDS for short, captures Bourbaki's technical notion adequately and allows us to extend it to categories and other structures that are inherently different from set-based structures. We then discuss why such a metamathematical analysis

ought to be taken seriously, at least as proper conceptual analysis, by anyone who wants to develop a philosophical stance towards structuralism.

\section{Discarding Bourbaki's Structuralism: A Prac- tical Guide}

Bourbaki's books have had from very early on a clear and undeniable influence on the mathematical community, on the development of mathematics, its expo- 
sition and its goals. It is extremely easy to find testimonies by mathematicians that attest this influence. Here is a sample:

All mathematicians of my generation, and even those of subsequent decades, were aware of Nicolas Bourbaki, the Napoleonic general whose reincarnation as a radical group of young French mathematicians was to make such a mark on the mathematical world. ... Many of us were enthusiastic disciples of Bourbaki, believing that he had reinvigorated the mathematics of the twentieth century and given it direction. $([1,1])$

Two elements have to be underlined in this quote. First, that many young mathematicians throughout the world considered themselves disciples of Bourbaki. This demonstrates Bourbaki's influence and impact. Second, Atiyah emphasizes the fact, and rightly so, that Bourbaki had given mathematics a direction. This has to be emphasized, for it indicates in what sense young mathematicians considered themselves disciples of Bourbaki. The French mathematician and philosopher of mathematics, Frédéric Patras, details this last claim as follows:

Mathematicians, like all scientists, need teleological purposes that allow them to structure and justify their discourse. Bourbaki had succeeded in giving an indisputable aura to mathematical thought, by giving it a spring - structuralism - and a finality - the search of a successful and hierarchical architecture of its concepts and results. ${ }^{1}$. $([33,119])$

In some sense - and this point would need to be developed, but I will not do so here - Bourbaki more or less consecrates the scientific character of mathematics by giving it a global, unified, theoretical organization, as opposed to a haphazard bunch of results, tools and methods. Mathematics's unity is revealed by abstract structures. These are ultimately what mathematics is about and what mathematicians should be looking for. Mathematical research, at least when it is concerned with the understanding of mathematics, aims at disclosing abstract structures, their properties and their combinations.

Despite this influence and despite the fact that, as we will see in the next section, Bourbaki defends and develops a thoroughly structuralist conception of mathematics, philosophers of mathematics have systematically ignored Bourbaki's work ${ }^{2}$. As we have already seen above, it is usually claimed that Bourbaki's work simply does not address the semantical, ontological and epistemological issues central to the contemporary philosophical disputes.

\footnotetext{
${ }^{1}$ Here is the original text in French: "Les mathématiciens, comme tous les scientifiques, ont besoin de vises téléologiques qui leur permettent de structurer et de légitimer leurs discours. Bourbaki avait réussi à donner une aura indiscutable à la pensée mathématique, en lui procurant un ressort - le structuralisme - et une finalité - la recherche d'une architecture aboutie et hiérarchisée de ses concepts et résultats."

${ }^{2}$ There is one important exception to this, namely the so-called structuralist school in philosophy of science of the 1970's and 1980's, led by Suppes, Suppe, Moulines and Balzer. Some of them explicitly based their work on Bourbaki's technical notion of structure. See, for instance, Erhard Scheibe's work in philosophy of physics.
} 


\subsection{Bourbaki's evaluation of his own analysis}

Bourbaki is partly responsible for this state of affairs, but for reasons that are different from those invoked by philosophers of mathematics. Bourbaki came to the conclusion that his original version of structuralism failed simply because it was, according to him, superseded by the notions of categories and functors. Again, here are typical claims made by, first, one of the founding fathers of Bourbaki and, second, by one of the most influential members of the second generation of Bourbaki.

Let us immediately say that this notion [that is, Bourbaki's notion of structure] has since been superseded by that of category and functor, which includes it under a more general and convenient form. It is certain that it will be the duty of Bourbaki, ..., to incorporate the valid ideas of this theory in his works. ([14]).

It is not that Bourbaki's analysis was wrong or useless, but simply that a better notion was available when the volume on structure finally came out. Pierre Cartier says essentially the same thing:

Bourbaki's volume on structure, which finally appears in 1957, includes all these evolutions. On the one hand, it is a shameful treatise on categories, where the key notions (categories, functor, etc., ...) appear in filigree, but not in the "official" text. On the other hand, it is a grammar of structures: analyzing his own style, Bourbaki describes a certain number of reasoning-types, which return whenever a certain structure shows up. Ironically, the science of structures does not go beyond the descriptive stage and does not access the structural stage. ([7, 22], my translation)

The fact is: Bourbaki did not know how to fit categories, functors, etc., in his general analysis of mathematical structures. This is deeply ironic for two reasons. First, it is undeniable that a category is a mathematical structure and, as such, it should be describable in Bourbaki's language of structures. Second, category theory can itself be used directly to describe mathematical structures and links between mathematical structures. According to Krömer's analysis of Bourbaki's archives on the subjects, Bourbaki envisaged adding a chapter of categories and functors after the chapter on structures. Somehow and for reasons that are not entirely clear, that chapter never made it to publication. (See [23, 143] for details.)

Be that as it may, Bourbaki came to the conclusion that his analysis of the general notion of abstract mathematical structure was somehow too short, for it did not encompass the notions of categories, functors, etc. This does not mean that Bourbaki concluded that his structuralist standpoint was refuted. Having worked on his volume on sets and structures for nearly 20 years and having published a series of influential books which were based on the latter, there were also practical reasons underlying his decision. Bourbaki could not rewrite all these 
volumes from a different starting point. Moreover, although categories, functors and natural transformations had been introduced in 1945 and used quickly after in algebraic topology and homological algebra, it was not a proper theory before the publication of Bourbaki's volume on sets and structures, namely 1957. Indeed, the concepts of adjoint functors and of equivalence of categories, to mention but the most two important notions, were introduced in print precisely in 1957 by Kan and Grothendieck respectively. Functor categories play a central role both in Kan's and in Grothendieck's works, thus raising pressing issues on the foundations of category theory itself. Grothendieck introduced the concept of representable functor only in 1961. Abstract categories, that is categories given by a list of axioms, for instance additive categories, abelian categories, etc. were also introduced at that time. The point is simply that when Bourbaki had finally decided to publish its volume on sets and structures, he only had an incomplete understanding of the nature and impact of category theory, both on mathematics and its foundations.

It would thus make sense to use Bourbaki's own evaluation of his work on the general notion of abstract mathematical structure to push it aside and adopt a categorical point of view, assuming that such an analysis is available. However, this is not what one finds in the philosophical literature. The reasons given in the latter are of a different nature altogether.

\subsection{The Philosophers' Evaluation of Bourbaki's Analysis}

To be fair, one has to remember that the contemporary philosophical discussion surrounding mathematical structuralism in the anglo-american world originated from Benacerraf's papers published in 1965 and 1973. In those papers, Benacerraf does not refer to Bourbaki. It is interesting to note, however, that Benacerraf's first paper opens up with a long quote taken from Richard Martin:

The attention of the mathematician focuses primarily upon mathematical structure, and his intellectual delight arises (in part) from seeing that a given theory exhibits such and such a structure... (...) But ... the mathematician is satisfied so long as he has some "entities" or "objects" (or "sets" or "numbers" or "functions" or "spaces" or "points") to work with, and he does not inquire into their inner character or ontological status. (R. M. Martin, quoted by $[2,272]$ )

Martin simply reflects the attitude adopted by the mathematical community, the structuralist stance that was pushed and developed by Bourbaki. Be that as it may, the fact is that most of the papers and books that have been written afterwards by philosophers were targeted at Benacerraf's arguments on truth and knowledge. Resnik is one of the first philosophers that have tried to develop a structuralist philosophy of mathematics. Nowhere in his writings does one find a reference to Bourbaki. In fact, Resnik even suggests to use the word "pattern" instead of the expression "structure" and qualify mathematics as the science of patterns. As Resnik readily admits, he is not so much interested in 
understanding structuralism as such, but rather he wants to defend and develop a form of (Quinean) realism about mathematics.

Basically the same can be said about Shapiro's well-known work on the subject. Shapiro has introduced what is now the standard terminology in the philosophical literature, namely the distinctions between ante rem structuralism, in re structuralism and modal structuralism. The philosophical literature focuses mostly on the qualifiers and less on structuralism as such. Like Resnik, Shapiro's goal is to articulate a form of structural realism about mathematical knowledge.

Interestingly enough, Shapiro does mention Bourbaki in his book [35]. He cites key passages from Bourbaki's The Architecture of Mathematics. However, at the end of the day, Shapiro relies on Leo Corry's evaluation of Bourbaki's contribution to mathematical structuralism.

Although their Theory of sets[1968] contains a precise mathematical definition of "structure", Corry [1992] shows that this technical notion play almost no role in the other mathematical work, and only a minimal role in the book that contains it. The notion of "structure" that underlies the work of Bourbaki and contemporary mathematics, is inherently informal... ([35, 177])

Shapiro quotes Corry at this point. We will reproduce only the last part of that quote, since it illustrates an important point that needs to be underlined.

[T] he rise of the structural approach to mathematics should not be conceived in terms of this or that formal concept of structure. Rather, in order to account for this development, the evolution of the nonformal aspects of the structural image of mathematics must be described and explained. ([10,342])

This book [namely Shapiro's book] is a contribution to the program described by that last sentence. $([35,342])$

Thus, Shapiro's work, as well as the work of many others, aims at describing and explaining the nonformal aspects of mathematical structures, since it is assumed that no such formal analysis can be provided. Thus, Bourbaki's work is irrelevant to the philosophical enterprise.

Hellman, in his work [19, 20], does not mention Bourbaki, although in [21], he considers category theory as a candidate for structuralism and, as such, could be said to be faithful to Bourbaki's spirit. However, he dismisses category theory as providing an autonomous framework for structuralism ${ }^{3}$.

Chihara in [8] does not refer to Bourbaki. The whole work is a conversation with Resnik, Shapiro, Hellman and others we have not mentioned. Finally, in

\footnotetext{
${ }^{3}$ To be fair, although Dieudonné and Cartier, as well as others, have claimed that category theory provided a more convenient framework for structural mathematics, to use Dieudonné terminology, he never offered a proper and general analysis of the notion of abstract mathematical structure in the language of categories nor can we find a clear claim made by a member of Bourbaki that the latter provides foundations for mathematics.
} 
his review of contemporary mathematical structuralism [9], J. Cole does mention Bourbaki in a footnote, and declares that Bourbaki defended a form of set-theoretical eliminative structuralism. Thus, Bourbaki is acknowledged, but confined to a precise type of mathematical structuralism ${ }^{4}$.

As we have seen, Leo Corry's analysis of Bourbaki's work played an important role in the recent literature on the subject. Corry has presented a detailed analysis of the evolution of the notion of structure in the first half of 20th century mathematics and has given a thorough and documented analysis of Bourbaki's work $^{5}$. In the end, and as we have already seen in Shapiro's quote, his evaluation of Bourbaki's notion of structure is critical and negative. We will restrict ourselves to a unique quote that, we believe, faithfully represents Corry's take on Bourbaki.

The central notion of structure, then, had a double meaning in Bourbaki's mathematical discourse. On the one hand, it suggested a general organizational scheme of the entire discipline, that turned out to be very influential. On the other hand, it comprised a formal concept that was meant to provide the underlying formal unity but was of no mathematical value whatsoever either within Bourbaki's own treatise or outside it. But Bourbaki's theory of structures ${ }^{6}$ was only one among several attempts to develop a general mathematical theory of structures, ... . $([13,32])$

Corry makes three distinct claims in this passage. First, structuralism as an organizational scheme was put forward by Bourbaki from very early on and was very influential. Everybody agrees that this is essentially correct. Second, the technical notion presented by Bourbaki was simply worthless, even for Bourbaki. Corry is far from being the only one saying this. Saunders Mac Lane, one of the creators of category theory, went so far as to say that Bourbaki's presentation "is the ugliest piece of writing to have come from Bourbaki's pen"." [24, 5]. This assertion can only be made if one takes Bourbaki's analysis as being essentially mathematical and not, as it was, metamathematical. Third, Bourbaki's technical notion is but one among many and one that is particularly bad. Corry's general position with respect to a technical notion of structure is that such a notion simply cannot exists, for the notion of structure has evolved and will always evolve beyond the boundaries of a formal analysis. It is worth giving another explicit quote on this particular point:

I will claim [in the article] that the "structural character of contemporary mathematics" denotes a particular, clearly identifiable way

\footnotetext{
${ }^{4}$ As we will see, this is not unfounded and unjustified.

${ }^{5}$ The main source here is his book: [11]. We agree with many claims made by Corry, in particular the claim that the volume on set theory stands apart in Bourbaki's output, and not because of its clarity and rigor. We disagree on one particular, but important point about Corry's approach, as we will make clear.

${ }^{6}$ When Corry uses the italics, he refers to the technical notion of structure.

${ }^{7}$ Note that this is an aesthetic evaluation. Elsewhere, Mac Lane called it "a cumbersome piece of pedantry." ([25, 181]). This is also an aesthetic judgment. It might nonetheless still be essentially correct, despite being ugly.
} 
of doing mathematics, which can however only be characterized in nonformal terms. After that specific way of doing mathematics was crystallized and became accepted in the 1930s, diverse attempts were made to prodide a formal theory within the framework of which the nonformal idea of "mathematical structure" might be mathematically elucidated. Many confusions connected to the "structural character of mathematics" arise when the distinction between the formal and the nonformal senses of the word is blurred. ([10, 316])

We fundamentally disagree with Corry on this claim. He is partly right when he claims that Bourbaki's influence was not a consequence of his technical notion of structure. But only partly so. It is always dangerous to claim that a way of doing mathematics can only be characterized in nonformal terms. We believe that Bourbaki's proposal contains the main ingredients of a formal analysis and that Makkai's FOLDS provides a completely general formal analysis of what structuralism means for abstract mathematics. Notice how Corry's claims resemble Burgess's evaluation quoted at the beginning or this paper. Corry recognizes the influence Bourbaki had within the mathematical community, but that influence had nothing to do with the technical notion of structures expounded by Bourbaki in his volume on set theory. This is indeed partly correct, to the extent that Bourbaki's formal notion of structure did not have a direct impact. However, a certain component of his notion did have a tremendous impact. We will get back to this point in due course.

This is how Bourbaki's analysis is dismissed and, in the end, ignored.

\section{Mathematical Structuralism: its architecture}

\subsection{Structuralism and the axiomatic method}

Before we look at the specific formal analysis presented by Bourbaki, we believe it is worth our while to rehearse some of the claims he made in the paper The Architecture of Mathematics, originally published in French in 1948, and then translated into English in 1950. For in the heads of Bourbaki's members, structuralism is more than the claim that abstract structures are what mathematics is about.

Of course, it includes the latter claim. As Bourbaki asserts himself in a footnote of that paper: "From this new point of view, mathematical structures become, properly speaking, the only "objects" of mathematics." $[3,11$, footnote †]. Notice that structuralism is not taken as a starting point. It follows from a "new point of view", with an emphasis on the novelty of the method here. Bourbaki clearly claims that its structuralist stance is a consequence of the axiomatic method. It is crucial to understand what he meant by the latter and how he saw its function or what its role is in the organization and development of mathematics.

Bourbaki has thoroughly assimilated Hilbert's and his school's directives to use the axiomatic method systematically. He also adopted the idea that the 
whole of mathematics could be based on the principles of set theory and that the latter could and should be presented in an axiomatic fashion ${ }^{8}$. Needless to say, this does not mean that doing mathematics merely amounts to deriving theorems from the axioms of set theory. Let us quote Henri Cartan, one of the founding members of Bourbaki, who published a paper in 1943 and which can be read as a companion to The Architecture of Mathematics.

Let us suppose that these axioms [of set theory] are chosen once and for all. Our mathematical theory cannot limit itself to be a dreary compilation of truths, ... . For mathematics to be an efficient tool and, for us, mathematicians, to be really interested in it, it must be a living construction; one must clearly see the sequence of theorems, group partial theories. ([5, 11] [our translation])

How does one see "the sequence of theorems, group partial theories"? With the help of ideas, concepts. According to Bourbaki, concepts constitute the core of mathematics and concepts are captured by the axiomatic method. To wit:

What the axiomatic method sets as its essential aim, is exactly that which logical formalism by itself cannot supply, namely the profound intelligibility of mathematics. [...] Where the superficial observer sees only two, or several, quite distinct theories, ..., the axiomatic method teaches us to look for the deep-lying reasons for such a discovery, to find the common ideas of these theories, buried under the accumulation of details properly belonging to each of them, to bring these ideas forward and to put them in their proper light. $([3,223])$

The last sentence clearly indicates what the axiomatic method is used for: to abstract common ideas from different theories. Thus, one could and should perhaps talk about the abstractmethod in this particular case ${ }^{9}$. The emphasis is on how mathematics develops, not on how it is founded or justified.

Thus that the axiomatic method is used to study the relations existing between different mathematical theories, hence to abstract from these mathematical theories. It is therefore grounded in classical mathematics, has its roots in the latter, it is nourished by its elements. But it yields new fruits, fruits that are of a different type than the soil it comes from. And in turn, the abstract structures reorganize the landscape completely: the seeds produce new fields, new crops $^{10}$.

\footnotetext{
${ }^{8}$ Bourbaki's axiomatic set theory was definitely odd, idiosyncratic and arguably inadequate. The details of Bourbaki's set theory has no impact on our main claim. However, for more on Bourbaki's set theory, see for instance [31].

${ }^{9}$ I have looked at this method in more details in the following two papers: [28], [29].

${ }^{10}$ It has to be pointed out that when Bourbaki writes and decides to systematically develop this standpoint, he finds resistance among contemporary mathematicians who believed that the axiomatic method was unable to produce any genuinely new concepts and results. At the risk of repeating ourselves, Bourbaki sees the axiomatic method as a creative method in mathematics. For a discussion of the creative role of axioms in mathematics, see also [34].
} 
From the axiomatic point of view, mathematics appears thus as a storehouse of abstract forms - the mathematical structures; (...) It is only in this sense of the work "form" that one can call the axiomatic method a "formalism". The unity which it gives to mathematics is not the armor of formal logic, the unity of lifeless skeleton; it is the nutritive fluid of an organism at the height of its development, the supple and fertile research instrument to which all the great mathematical thinkers since Gauss have contributed, all those who, in the words of Lejeune-Dirichlet, have always labored to "substitute ideas for calculations". ([3, 231]

These forms are not generated randomly, nor are they mere generalizations. They are original ideas in both sense of the word: not only are they new, but in the logical order, the classical ideas are special cases of these innovations, and in that sense, the classical ideas originate from them. Furthermore, at the time, Bourbaki believed that three families of basic forms could be identified, families whose surname started with a 'c': composition, continuity and comparison, corresponding respectively to algebraic structures, topological structures and order structures. He even suggested they be called "mother structures".

\subsection{Mother Structures and Their Descendants}

It is important to understand the organization of mathematics that naturally follows from Bourbaki's usage of the axiomatic method. First, the mother structures are seen as tools:

It should be clear from what precedes that its most striking feature [of the axiomatic method] is to effect a considerable economy of thought. The "structures" are tools for the mathematician; as soon as he has recognized among the elements, which he is studying, relations which satisfy the axioms of a known type, he has at his disposal immediately the entire arsenal of general theorems which belong to the structure of that type. $([3,227])$

This probably sounds entirely trivial today, but was not when Bourbaki was writing. One of the byproducts of this economy of thought is that the solution to certain problems do not depend on the personal talent of a mathematician. The latter now has a toolbox of concepts, theories, results that can be applied directly to various cases, apparently unrelated. One only needs to verify that this particular problem is indeed a case of the abstract structure and apply the relevant theorems to the problem at hand.

Thus, these mother structures modify the very practice of mathematics. But they also transform the very fabric of mathematics.

In place of the sharply bounded compartments of algebra, of analysis, of the theory of numbers, and of geometry, we shall see, for example, that the theory of prime numbers is a close neighbor of the theory of 
algebraic curves, or, that Euclidean geometry borders on the theory of integral equations. The organizing principle will be the concept of a hierarchy of structures, going from the simple to the complex, from the general to the particular. $([3,228])$

One starts from the most general, the simplest, in other words the structures with the smallest number of axioms. It is then possible to add axioms to these mother structures to obtain more specific structures, e.g. Hausdorff topological spaces, uniform spaces, abelian groups, linearly ordered sets, etc. Thus, each mother structure already has an impressive family tree.

Of course, these structures also combine together, these combinations yielding more than the simple addition of the original structures.

Beyond the first nucleus, appear the structures which might be called multiple structures. They involve two or more of the great mother structures simultaneously not in simple juxtaposition (which would produce nothing new), but combine organically by one or more axioms which set up a connection between them. ([3, 229])

Bourbaki exemplifies the latter by comparing topological algebra to algebraic topology ${ }^{11}$. We are still at the level of abstract structures, although no longer in the original trees of the mother structures. It is possible to go down further still and end up working on the classical structures of mathematics, for instance the real numbers ${ }^{12}$.

Farther along we come finally to the theories properly called particular. ... At this point we merge with the theories of classical mathematics, the analysis of functions of a real or complex variable, differential geometry, algebraic geometry, theory of numbers. But they have no longer their former autonomy; they have become crossroads, where several more general mathematical structures meet and react upon one another. $([3,229])$

There are numerous epistemological gain to this way of working. We have already mentioned simplicity. Cartan is a bit more explicit about these in his paper:

Thus, not only the axiomatic method, based on pure logic, gives an unshakable basis to our science, but it allows us to better organize

\footnotetext{
${ }^{11}$ This is a point where the introduction of category theory would have changed the picture considerably. Indeed, a whole section should be written on the development of the axiomatic method in the language of categories, as was done by Grothendieck in his paper on homological algebra [16]. It can be argued that Grothendieck's way of doing mathematics is a natural extension of Bourbaki's presentation in The Architecture of Mathematics. Thus, one aspect of Grothendieck's style is not that surprising when seen in this light.

${ }^{12} \mathrm{We}$ are not saying that this is original with Bourbaki. The idea, at least restricted to algebra, was already implicit in van der Waerden's Moderne Algebra and explicit in a paper written by Helmut Hasse in 1931. (See [18].) Since many members of Bourbaki have worked with Hasse and others of the German school in these years, it is not entirely ridiculous to believe that they had discussed these matters with them.
} 
it and to better understand it, it makes it more efficient, it substitute general concepts to "computations", which, done haphazardly, would likely lead to nothing, unless done by an exceptional genius. $([5,11])[$ our translation]

Better organization, better understanding, more efficiency are obvious epistemological virtues. Better understanding is obtained by the separation of the abstract components involved in proofs. Indeed, it is possible to identify the role played by the various abstract components in a given proof.

\section{Metamathematical Structuralism: its nature}

As we have said, Bourbaki's presentation contains two components that are somehow confused, even by Bourbaki himself ${ }^{13}$. The first component can be presented in a purely mathematical manner if one wants, although Bourbaki himself does not. It is the notion of echelon of structure. The second component is clearly metamathematical and is a formal requisite on any mathematical theory that pertains to talk about abstract mathematical structures. Joined together they yield the notion of species of structure. Most of the literature and Bourbaki himself have concentrated on the first aspect, on the mathematical notion of structure. The metamathematical has been more or less evacuated. We want to reverse completely this tendency and put the metamathematical component at the forefront.

\subsection{Confusing the tree for the forest: the notion of math- ematical structure}

In The Architecture of Mathematics, Bourbaki gives an informal presentation of the notion of mathematical structure.

It can now be made clear what is to be understood, in general, by a mathematical structure. The common character of the different concepts designated by this generic name, is that they can be applied to sets of elements whose nature has not been specified; to define a structure, one takes as given one or several relations, into which these elements enter; then one postulates that the given relation, or relations, satisfy certain conditions. To set up the axiomatic theory of a given structure, amounts to the deduction of the logical consequences of the axioms of the structure, excluding every other hypothesis on the elements under consideration. ([3, 225-226])

\footnotetext{
${ }^{13}$ This claim might sound silly, but it is fairly easy to explain how it happened by looking at the Bourbaki archives and the evolution of the project. It has to be kept in mind that Bourbaki had no logician among its members. Claude Chevalley was the only founding member who was interested in logic and metamathematics and there is clear evidence that he was responsible for the presence of logic and the metamathematical standpoint in the various versions of the notion. Other members, like Dieudonné and Weil, thought that logic and metamathematics were peripheral and secondary to the whole enterprise.
} 
It is important to notice that Bourbaki attempts to give a totally general notion of mathematical structure. At the time, various particular cases were wellknown, leading Bourbaki to introduce the idea of mother structures in his popular paper ${ }^{14}$. To give a general analysis is another enterprise altogether, bound to be somewhat opaque and mysterious at first. It is, of course, the result of an analysis, which is done with the various examples in mind. One of the problems that Bourbaki encountered is that these examples kept popping up and kept being somewhat different from the original ones, forcing him to adjust his analysis - for instance with the case of modules which forced Bourbaki to introduce fixed parameters in the analysis -, and in the end, with the advent of categories, even to give it up!

The core of the analysis is simple enough ${ }^{15}$. We assume the language of first-order logic. Notwithstanding the cumbersome notation used by Bourbaki, the underlying ideas are simple enough ${ }^{16}$. One starts with a finite list of set variables, which we will denote by $\vec{A}=A_{1}, A_{2}, \ldots, A_{n}$ and a finite list of set constants, or parameters, which we will denote by $\vec{B}=B_{1}, \ldots, B_{m}$. Of course, in many cases, there are no parameters, but in others, they are indispensable, for instance for vector spaces, where the parameter is a field $k$, or modules, where the parameter is a ring $R$. Bourbaki first defines what he calls an echelon construction: it is a collection of $E$ of terms defined inductively by the following simple rules: 1 . each of $A_{1}, \ldots, A_{n}, B_{1}, \ldots B_{m}$ is in $E ; 2$. If $X$ and $Y$ are in $E$, so is $X \times Y$; 3 . If $X$ is in $E$, so is $\wp(X)$. Thus, an echelon construction $E$ provides us with all possible basic terms that are required for a structure of a given kind to be defined. This is how Bourbaki intends to cover all possible types of abstract structures to start with. Once this is done, it is necessary to introduce ways to restrict this echelon to get back to actual structures. For instance, for most algebraic structures, various products and powers of a certain type will be necessary, and for topological structures, powers, and powers of powers, etc., will be indispensable, and some other products and powers will be necessary to specify certain properties of the structure. Let us denote an element of an echelon construction $E$ by $S_{i}$ and we call such an element a sort.

Thus, given a echelon construction $E$, the next step is to pick elements $S_{1}, \ldots, S_{p}$ of $E$, which we can call specified sorts, that is those that are necessary for the definition of the type of structure one has in mind. One then adds a list $R_{1}, \ldots, R_{k}$ of sorted relation symbols ${ }^{17}$. This yields what is commonly

\footnotetext{
${ }^{14}$ The notion of mother structure is nowhere to be found in the official texts.

${ }^{15}$ We have to point out, as many have done, that Bourbaki's presentation of logic and set theory is very idiosyncratic and it is difficult to understand why he clung to his vocabulary and axioms. One obvious example is his choice to talk about assemblage to designate what any other logician calls a formula. Most commentators would focus on his choice of the $\tau$ operator and his axioms for set theory, and rightly so. He could easily have used standard notation and notions at that point, since after all, Kleene's monumental Introduction to Metamathematicswas published in 1952, to mention but the most famous textbook available at the time. Bourbaki was well aware of Hilbert \& Ackermann's book published in 1928, but he unfortunately did not adopt its conventions.

${ }^{16}$ We are not following Bourbaki's conventions, which we find unnecessarily complicated and we simplify both the notation and the presentation.

${ }^{17}$ This can easily be translated into purely formal requirements of the usual kind.
} 
called a signature or a similarity type $\mathcal{L}=\mathcal{L}(\vec{A}, \vec{B}, \vec{S}, \vec{R})$. By interpreting these symbols in the obvious way in the domain of sets, one obtains the notion of an $\mathcal{L}$-structure. We should hasten to add that this is not yet the notion of structure we are driving at, nor is it yet Bourbaki's notion. Bourbaki defines what he calls 'a species of structure' and for that a key component is still missing: the notion of isomorphism or, in Bourbaki's terminology, transport of relation.

Before we introduce it, it should be noted that in most presentations of Bourbaki's analysis, the distinction between the mathematical and the metamathematical levels is usually blurred. For instance, here is how Corry presents the notion of a species of structure in one of his articles:

Now to define a 'species of structure' $\Sigma$ we take:

1. $n$ sets $x_{1}, x_{2}, \ldots, x_{n}$; the 'principal base sets';

2. $m$ sets $A_{1}, A_{2}, \ldots, A_{m}$; the 'auxiliary base sets' and

3. a specific echelon construction scheme:

$$
S\left(x_{1}, \ldots, x_{n}, A_{1}, \ldots, A_{m}\right) .
$$

This scheme will be called the 'typical characterization of the species of structure $\Sigma$ '. The scheme is obviously a set and the structure is now defined by characterizing some of the members of this set by means of an axiom of the species of structure. This axiom is a relation which the specific member $s \in$ $S\left(x_{1}, \ldots, x_{n}, A_{1}, \ldots, A_{m}\right)$ together with the sets $x_{1}, \ldots, x_{n}, A_{1}, \ldots, A_{m}$ must satisfy. The relation in question is constrained to satisfy the conditions of what Bourbaki calls a 'transportable relation', which means roughly that the definition of the relation does not depend upon any specific property of $s$ and the sets in themselves, but only refers to the way in which they enter in the relation through the axiom. ([10, 323-324])

Notice how Corry presents everything directly in terms of sets. This simply reads like we are dealing with a generalization of the presentation of any usual mathematical structure, e.g. the group structure, the topological structure, etc. We have sets, certain basic set-theoretical operations on them necessary to define specific relations and operations on them and, finally, axioms specifying the specific properties that these relations have to satisfy. Except for the following bit on transportable relation, which is not explained and seem simple enough. It is very easy to lose track that we are firmly in a metamathematical context.

The fact that in the volume on sets, the chapter immediately preceding the section on structure treats the notions of ordered sets, cardinals and integers does not help. We are squarely in set theory. The reader is thus asked to move back to the metamathematical mode without any specific warning at this moment. Furthermore, the foregoing presentation can, in fact, be presented directly 
in terms of sets and operations on sets, functions on sets, etc ${ }^{18}$. In some of the earlier versions of the chapter on structures, Bourbaki himself does not carefully make the distinction. In the final volume, we are clearly in metamathematics, but the presentation oscillates between sets and formal expressions. What one has to understand, is that Bourbaki also introduces a completely general notion of isomorphism between instances of a species of structure and that the latter determines directly and precisely what one can write down and prove about these structures. And this, in our mind, is the key element.

\subsection{Seeing the Forest: The Notion of Isomorphism}

In the same way that Bourbaki had to give a completely general notion of structure, he had to give a completely general notion of isomorphism of structure as well. At the time, once again, numerous particular examples were clear: various specific notions of isomorphism for algebraic structures were known, similarly for topological structures and order structures. It should be emphasized that the identification of the correct notion of isomorphism for a structure of a given type is not a trivial business and that, in some cases, it took quite some time before the community of mathematicians finally settled on the right notion ${ }^{19}$. It also has to be pointed out that there is no mention of isomorphism in The Architecture of Mathematics. This omission is perhaps attributable to the fact that the latter paper was aimed at a general audience and that Dieudonné might have felt that explaining even particular cases of isomorphisms would simply be too technical in such a short paper ${ }^{20}$. A quick examination of the Bourbaki archives shows clearly that the notion of isomorphism was nonetheless incorporated in the analysis right from the start. Ten years after The Architecture of Mathematics, when Cartan reflects on Bourbaki, the emphasis is crystal clear:

Bourbaki's decision to use the axiomatic method throughout brought with it the necessity of a new arrangement of mathematics' various

\footnotetext{
${ }^{18} \mathrm{It}$ is, indeed, very tempting to start the analysis in the category of sets and define the notion of species of structures directly there. That would yield a perfectly acceptable mathematical analysis of that latter notion. It is probably what Bourbaki would have done, had he agreed on a way to do it. It was done by Ehresmann in [15] and, more recently and in a different context, by Joyal in [22]. Our goal, and we believe Bourbaki's goal too, is to provide a genuine metamathematical analysis, something that is required to anchor a structuralist standpoint about the whole of abstract mathematics.

${ }^{19}$ Two specific and surprising cases have to be mentions: the notion of homeomorphism for topological spaces and the notion of equivalence of categories. In the case of topological spaces, mathematicians did not see immediately what the right notion was and there was some confusion in the literature for quite some time. See [32] for details. As for categories, Eilenberg and Mac Lane introduced the notion of isomorphism of categories in 1945, thinking that it was the proper criterion of identity for them. The right notion, namely the notion of equivalence, was introduced by Grothendieck in his paper in homological algebra in 1957, thus twelve years after the publication of Eilenberg and Mac Lane's original paper. See [27].

${ }^{20}$ Dieudonné does not give the definition of a topological space either, believing that "the degree of abstraction required for the formulation of the axioms of such a structure is decidedly greater that it was in the preceding examples; the character of the present article makes it necessary to refer interested readers to special treatises" ([3, 227].
} 
branches. It proved impossible to retain the classical division into analysis, differential calculus, geometry, algebra, number theory, etc. Its place was taken by the concept of structure, which allowed the definition of the concept of isomorphism and with it the classification of the fundamental disciplines within mathematics. $([6,177])$

It is remarkable to see that the concept of structure allows the definition of isomorphism. They go hand in hand. The fact that they form a pair is obfuscated by the emphasis on the axiomatic method. But in Bourbaki's presentation, the notion of isomorphism is an intrinsic part of the axiomatic method. It should also be pointed out that the title of the first section of the fourth chapter is 'Structures and isomorphisms'.

As we have seen in Corry's presentation, the transport of relation - which is quickly shown by Bourbaki to be equivalent to the notion of isomorphism - is often thought of as a constraint which merely guarantees that, to quote Corry again, "the definition of the relation does not depend upon any specific property of $s$ and the sets in themselves, but only refers to the way they enter in the relation through the axiom ${ }^{21}$. Of course, this is entirely correct, and as a comment aimed at expressing a key feature of transport of relations, it is entirely acceptable. However, it is necessary to be a little more precise here.

The crucial element is that transportable relations are built-in Bourbaki's notion of a theory of a species of structure. This means, literally, that the only theorems provable in a theory of a species of structure are those that are invariant under isomorphism.

Bourbaki proceeds as follows. We go back to our basic set variables, but we now suppose that we have two lists of terms $\vec{A}=A_{1}, A_{2}, \ldots, A_{n}$ and $\overrightarrow{A^{\prime}}=A_{1}^{\prime}, A_{2}^{\prime}, \ldots, A_{n}^{\prime}$. We now add terms $f_{1}, \ldots, f_{n}$ to our theory such that the relations " $f_{i}$ is a function from $A_{i}$ to $A_{i}^{\prime}$ " are theorems of the theory, for all $1 \leq i \leq n$. It can then be shown that these $f_{i}$ s can be canonically extended to the echelon construction. Moreover, if the original $f_{i}$ s are bijections, the canonical extensions are bijections too ${ }^{22}$. We can now introduce the notion of transport of structure.

We have our specified sorts and sorted relations. For a relation $R$ to be transportable in a theory $T$ in the language $\mathcal{L}$ means that it can be proved in the theory $T$ that the relation $R$ holds for the sorts defined over $\vec{A}_{i}$ if and only if $R$ holds for the sorts over $f_{i}\left(A_{i}\right)=A_{i}^{\prime}$ and their canonical extensions in the echelon construction. Again, this claim can be made very precise with the proper notation, but it would occupy an unnecessarily long portion of this

\footnotetext{
${ }^{21}$ Corry is very well aware of the fact that there were no general analysis of the notion of isomorphism before Bourbaki. Indeed, in a different paper, he says "None of these concepts, however, is defined in a general fashion so as to be a priori available for each of the particular algebraic systems [in van der Waerden's textbook]. Isomorphisms for instance, are defined separately for groups and for rings and fields, and van der Waerden showed in each case that the relation "is isomorphic to" is reflexive, transitive and symmetric" $([12,172]$.

${ }^{22}$ Notice the ambiguity here. We are still in metamathematics, but it is all too easy to fall back on a purely set theoretical reading of the definitions and notions given. We are talking about set theoretical formulas all along. We have to write down formulas such that, when they are interpreted in sets, then the $f_{i}$ s are bijections.
} 
paper. The crucial point to notice is the fact that one has to prove in the theory that the relations are transportable and that the notion of species of structure has not been defined yet. We are now ready to do so, for Bourbaki requires that the relations used in the axioms of a structure provably be transportable.

Bourbaki is very clear: a species of structure $\Sigma$ in the language of set theory is a text, i.e. a series of first-order formulas of set theory in the given signature $\mathcal{L}$ together with a transportable relation $R$, the latter called the axiom of the species of structure ${ }^{23}$. In a terminology which is more in tune with contemporary logical conventions, one can say that a Bourbaki species of structure is given by the $\mathcal{L}$-structures whose relations are transportable, in other words, as Bourbaki himself shows, whose relations satisfy the condition of isomorphism invariance. Thus, Bourbaki incorporates in the axiomatic method itself the requirement that the defining properties of a structure be invariant under isomorphism. It is primordial to understand that the latter is a metamathematical requirement, in the sense that it has to be provable in the theory that the relation is invariant under isomorphism. And this gives us what deserves to be called the structuralist motto: for a logical theory to be considered a structuralist theory for abstract mathematics, it has to satisfy the following property: for objects $X$ and $Y$ of the theory $\mathcal{T}$, the only properties $P$ that are legitimate in $\mathcal{T}$ are those that satisfy invariance under isomorphism: If $P(X)$ and $X \cong Y$, then $P(Y)$. Clearly, Bourbaki's development of mathematics satisfies this motto.

Remember that the volume on set theory is the only volume in which Bourbaki takes a metamathematical standpoint. When one looks at the other volumes, say on topology or algebra, the definitions proceed in the standard fashion. Bourbaki does not state that the axioms of a topological space are given by a text or by formulas in the language of set theory. He simply states them. But what he does, and in fact systematically, is to introduce the proper notion of isomorphism by invoking the general construction given in the chapter on structures ${ }^{24}$. The general notion of isomorphism is used as it should be in the remaining volumes. And that is the whole point. This is why and how one knows what is, for instance, a topological property or a group property, etc. And this is precisely why mathematicians do not fall prey to Benacerraf's problem.

It is striking to see that the remaining sections of the chapter on structures reads more and more as a standard mathematical text and less and less as

\footnotetext{
${ }^{23}$ This is very surprising. Of course, Bourbaki does not literally mean that a species of structure is a text, for it has to be an interpretation of that text in a mathematical domain. There is no doubt that Bourbaki understood that, but this is what one reads. I suspect that then emphasis on the text was deliberate in order to emphasize the metamathematical nature of the analysis.

${ }^{24}$ See, for instance definition 3 in the first section of the volume on general topology. The reference is explicit. The definition given by Bourbaki of isomorphism of topological spaces is not the standard definition. Bourbaki then immediately shows that the definition that follows from the general notion of isomorphism of structure is equivalent to the standard definition of homeomorphism. Corry is absolutely correct to point out that "the verification of this simple fact (which is neither done nor suggested in the book) is a long and tedious (though certainly straightforward) formal exercice" $([10,330])$. However, it misses the main point, which is essentially metamathematical.
} 
a metamathematical analysis. It is also striking that the notions presented in the published version are indeed concepts that are afterwards presented in the language of categories: initial structure, product structure, final structure, quotient structure and universal map. And even as such, the presentation is clumsy. But as we have said, had Bourbaki decided to present these ideas in the language of categories, he would probably not have done a better job, since the latter had not reached its maturity in the mid $1950 \mathrm{~s}^{25}$.

\section{FOLDS: encompassing all forms of structures}

Bourbaki's structuralism faced two difficulties. First, the theory one starts with, namely set theory, is not itself structural. This might or might not be a serious problem, but it certainly deserves a discussion. For one thing, the question whether it is possible to build a set theory that would satisfy the structuralist motto is worth investigating. Second, as we have already mentioned, categories do not fit easily and immediately in Bourbaki's scheme. The problem arises with the very definition of a category. Is a category a set? Clearly, the category of all set cannot be a set. This was the first problem faced by Bourbaki (and, in fact, all those using categories). Second, the criterion of identity for categories is given by the notion of equivalence of categories, not the notion of isomorphism. Bourbaki could not have known this when he published his volume on sets and structures, for the notion of equivalence of categories was introduced by Grothendieck in 1957. Thus, the notion of category raises a new metamathematical challenge, for the notion of transport of relation as given by Bourbaki is inadequate for categories.

Both of these problems now have a solution that is consistent with the structuralist motto. These solutions rely on the logical framework developed by Michael Makkai more than twenty years ago, namely First-Order Logic with Dependent Sorts, or FOLDS for short ${ }^{26}$.

It is impossible to do justice to FOLDS in such a short paper. We will summarize its mains features and emphasize how it captures the fundamental idea underlying Bourbaki's approach and how to generalize it to more abstract forms. First, FOLDS is a revision of first-order logic. For one thing, it is a multisorted syntax. Second, these sorts are dependent sorts. For instance, when one writes $f: X \rightarrow Y$, the underlying syntax is that one has $X, Y:$ Object, that is

\footnotetext{
${ }^{25}$ Thus, we disagree with Corry's evaluation that by 1957 , category theory had reached the status of an independent discipline that enabled generalized formulations of several recurring mathematical situations. Mac Lane had further developed some central ideas in his article on 'duality' ([10, 332]). Category was not yet an independent discipline and although it did enabled generalized formulations of several recurring mathematical situations, these were restricted to algebraic topology and homological algebra. Mac Lane's paper was not very influential and it is with hindsight that one sees into it some of the ideas that will become central after 1957 , once they will be shown to be systematically related to central concepts of the theory.

${ }^{26}$ Makkai published only one "official" paper on FOLDS, namely [26]. There is much more available on his web site: http://www.math.mcgill.ca/makkai/. For an informal presentation of FOLDS and some aspects of its motivation, see [30].
} 
$X$ and $Y$ are declared to be variables of sort 'Object' and then $f: \operatorname{Arr}(X, Y)$ is declared to be a variable of sort 'Arrow' and $f$ depends on $X$ and $Y$. One has to provide syntactical rules, which will necessarily be more complicated than the usual syntactical rules for first-order logic, for such a system and it is precisely what is done in FOLDS.

FOLDS has to be seen as a general theory of mathematical identity. Indeed, in FOLDS, mathematical identity is not given a priori and it is not a relation, it is derived from a given signature in the language and it is a structure. Thus, when FOLDS is used to develop set theory, the criterion of identity is not given by the axiom of extensionality, but by the structure between functions that defines the notion of bijections between sets. When FOLDS is used to develop (1-)category theory, one gets the notion of equivalence of categories. When FOLDS is used to develop bicategories, the notion of isomorphism one gets is the notion of biequivalence of categories. When FOLDS is used to develop homotopy theory, one gets the notion of homotopy equivalence.

As a consequence of the way it handles identity, that is the notion of 'isomorphism' for the type of structure obtained from a signature, it is possible to prove that the invariance principle holds: given a language $\mathcal{L}$ with its notion of $\mathcal{L}$-isomorphism and $\mathcal{L}$-structures $M$ and $N$, then if $\vDash_{M} \phi$ and $M \simeq_{\mathcal{L}} N$, then $\vDash_{N} \phi$. It turns out that Bourbaki's way of dealing with invariance of isomorphism can be described in this set up. Thus we believe that FOLDS does provide a formal, metamathematical analysis of what it is to be an abstract mathematical structure.

\section{From Metamathematical Structuralism to Philo- sophical Structuralism}

If structuralism for abstract mathematics is to hold any water, it ought to be based on a metamathematical analysis that captures the fundamental intuition underlying it. I claim that this is precisely what FOLDS provides.

In contrast with what one finds in the paper The Architecture of Mathemat$i c s$, we do not end up with three kinds of mother structures. This is not the point and there is nothing in the framework itself that points towards some privileged structures. We have a completely general formal framework that allows us to see how a purely structural mathematical framework can be developed. It does indicate an architecture of mathematics, how certain abstract structures are build one upon others. Furthermore, it is clearly open ended, with levels of abstractions and interplays between and within these levels. In a way, Bourbaki already had a glimpse of the evolution of species of structures.

It is quite possible that the future development of mathematics may increase the number of fundamental structures, revealing the fruitfulness of new axioms, or of new combinations of axioms. We can look forward to important progress from the invention of structures, by considering the progress which has resulted from actually known 
structures. On the other hand, these are by no means finished edifices; it would indeed be very surprising if all the essence had already been extracted from the principles. $([3,230])$

Thus, Bourbaki certainly envisaged the possibility that new fundamental structures might emerge. What he did not see coming was the possibility that these new structures would be more abstract than the ones he was familiar with ${ }^{27}$.

How is this related to philosophical structuralism? I believe that it is directly related to it. The usual questions about reference, meaning and truth, for instance, can now be put in their proper formal context. The epistemological and the ontological issues can also be formulated in the proper framework. As a bonus, mathematical structuralism and philosophical structuralism are now aligned along the same lines. This is what foundational research is all about: revealing explicitly what underlies the practice of a kind of mathematics and allowing for a better reflection of the philosophical content of that practice.

\section{References}

[1] Michael Atiyah. Bourbaki, A Secret Society of mathematicians and The Artist and the Mathematician-A Book Review. Notices of the AMS, 54(9):1150-1152, 2007.

[2] Paul Benacerraf. What numbers could not be. The Philosophical Review, 74(1):47, Jan 1965.

[3] Nicolas Bourbaki. The architecture of mathematics. American Mathematical Monthly, 57(221-232), 1950.

[4] John P. Burgess. Rigor and structure. Oxford University Press, Oxford, 2015.

[5] Henri Cartan. Sur le fondement logique des mathématiques. Revue scientifique, LXXXI:3-11, 1943.

[6] Henri Cartan. Nicolas Bourbaki and contemporary mathematics. Math. Intelligencer, 2(4):175-180, 1979/80.

[7] P Cartier. Le structuralisme en mathématiques: mythes ou réalité? Technical report, Bures-sur-Yvette, April 1998.

[8] Charles S. Chihara. A structural account of mathematics. The Clarendon Press, Oxford University Press, New York, 2004.

\footnotetext{
${ }^{27}$ One of the things that Bourbaki did not see is the importance of the relations between structures, in particular between algebraic and topological structures, which are made explicit and possible by category theory. The most striking example of that interaction that was available from early on is the equivalence between the category of affine schemes and the (opposite) of the category of commutative rings. It is this interaction that allowed Grothendieck to develop new foundations for algebraic geometry, foundations that are not given by the axiomatic method.
} 
[9] Julian C. Cole. Mathematical structuralism today. Philosophy Compass, 5(8):689-699, 2010.

[10] Leo Corry. Nicolas Bourbaki and the concept of mathematical structure. Synthese, 92(3):315-348, 1992.

[11] Leo Corry. Modern algebra and the rise of mathematical structures, volume 17 of Science Networks. Historical Studies. Birkhäuser Verlag, Basel, 1996.

[12] Leo Corry. Mathematical structures from hilbert to bourbaki: the evolution of an image of mathematics. In A. Bottazzini, U. \& Dahan Dalmedico, editor, Changing Images in Mathematics: From the French Revolution to the New Millenium, Studies in the History of Science, Technology and Medicine, pages 167-186. Routledge, New York, 2001.

[13] Leo Corry. Writing the ultimate mathematical textbook: Nicolas bourbaki's éléments de mathématique. In E Robson and J Stedall, editors, The Oxford Handbook of the History of Mathematics, pages 565-588. Oxford University Press, Oxford, 2009.

[14] Jean A Dieudonné. The Work of Nicholas Bourbaki. The American Mathematical Monthly, 77(2):134, February 1970.

[15] Charles Ehresmann. Catégories et structures. Dunod, Paris, 1965.

[16] Alexander Grothendieck. Sur quelques points d'algèbre homologique. Tôhoku Math. J. (2), 9:119-221, 1957.

[17] Ian Hacking. Why is there philosophy of mathematics at all? Cambridge University Press, Cambridge, 2014.

[18] Helmut Hasse. The modern algebraic method. The Mathematical Intelligencer, 8(2):18-23, January 1986.

[19] Geoffrey Hellman. Mathematics without numbers: Towards a modalstructural interpretation. Clarendon Press, 1989.

[20] Geoffrey Hellman. Structuralism without Structures. Philosophia Mathematica. Series III, 4(2):100-123, 1996.

[21] Geoffrey Hellman. Does Category Theory provide a Framework for Mathematical Structuralism? 11(2):129-157, 2003.

[22] André Joyal. Une théorie combinatoire des séries formelles. Advances in Mathematics, 42(1):1-82, 1981.

[23] Ralf Krömer. La "machine de grothendieck" se fonde-t-elle seulement sur des vocables métamathématiques? bourbaki et les catégories au cours des années cinquante. Revue d'histoire des mathématiques, 12:119-162, 2006. 
[24] Saunders Mac Lane. Letters to the editor. The Mathematical Intelligencer, 8(2):5-5, Jun 1986.

[25] Saunders Mac Lane. Structure in Mathematics. Philosophia Mathematica. Series III, 4(2):174-183, 1996.

[26] Michael Makkai. Towards a categorical foundation of mathematics. In J.A. Makowsky and E.V. Ravve, editors, Logic Colloquium '95 (Haifa), volume 11 of Lecture Notes in Logic, pages 153-190. Springer Verlag, Berlin, 1998.

[27] Jean-Pierre Marquis. From a Geometric Point of View: a study in the history and philosophy of category theory, volume 14 of Logic, Epistemology, and the Unity of Science. Springer, 2009.

[28] Jean-Pierre Marquis. Mathematical abstraction, conceptual variation and identity. In P-E Bour; G. Heinzmann; W. Hodges; P. Schroeder-Heister, editor, Logic, Methodology and Philosophy of Science, proceedings of the fourteen international congress, pages 299-322, London, 2015. College Publications.

[29] Jean-Pierre Marquis. Stairway to Heaven: The Abstract Method and Levels of Abstraction in Mathematics. The Mathematical Intelligencer, 38(3):4151, August 2016.

[30] Jean-Pierre Marquis. Unfolding folds: A foundational framework for abstract mathematical concepts. In Elaine Landry, editor, Categories for the Working Philosophers, pages 136-162. Oxford University Press, 2018.

[31] A R D Mathias. Hilbert, Bourbaki and the Scorning of Logic. In Infinity and truth, pages 47-156. World Sci. Publ., Hackensack, NJ, 2014.

[32] Gregory H. Moore. The evolution of the concept of homeomorphism. Historia Math., 34(3):333-343, 2007.

[33] Frédéric Patras. La pensée mathématique contemporaine. PUF, 2001.

[34] Dirk Schlimm. On the creative role of axiomatics. The discovery of lattices by Schröder, Dedekind, Birkhoff, and others. Synthese, 183(1):47-68, 2011.

[35] Stewart Shapiro. Philosophy of mathematics. Oxford University Press, New York, 1997. Structure and ontology. 